\title{
Communication
}

\section{In-Situ Depth Measurement of Laser Micromachining}

\author{
Xiaoming Chen ${ }^{1}\left(\mathbb{D}\right.$, Ying $\mathrm{Xu}^{1}{ }^{1}$, Nan-Kuang Chen ${ }^{2}$, Shannon Shy ${ }^{3}$ and Hsiang-Chen Chui ${ }^{1,3, * \mathbb{C}}$ \\ 1 School of Optoelectronic Engineering and Instrumentation Science, Dalian University of Technology, \\ Dalian 116024, China; chen_xm@dlut.edu.cn (X.C.); 32042038@mail.dlut.edu.cn (Y.X.) \\ 2 School of Physics Sciences and Information Technology, Liaocheng University, Liaocheng 252000, China; \\ nankuang@gmail.com \\ 3 Department of Photonics, National Cheng Kung University, Tainan 70101, Taiwan; ss710892@gmail.com \\ * Correspondence: hcchui@dlut.edu.cn
}

check for updates

Citation: Chen, X.; Xu, Y.; Chen, N.-K.; Shy, S.; Chui, H.-C. In-Situ Depth Measurement of Laser Micromachining. Photonics 2021, 8 , 493. https://doi.org/10.3390/ photonics 8110493

Received: 17 September 2021

Accepted: 3 November 2021

Published: 4 November 2021

Publisher's Note: MDPI stays neutral with regard to jurisdictional claims in published maps and institutional affiliations.

Copyright: (c) 2021 by the authors. Licensee MDPI, Basel, Switzerland. This article is an open access article distributed under the terms and conditions of the Creative Commons Attribution (CC BY) license (https:// creativecommons.org/licenses/by/ $4.0 /)$.

\begin{abstract}
Precision laser micromachining plays an important role in the biomedical, electronics, and material processing industries. During laser drilling, precision depth detection with micrometer-level resolution is required, particularly with blind-hole or heterogeneous structures. We present an optical detection system utilizing an optical confocal structure, experimentally confirmed to achieve a $>95 \%$ accuracy for micron-diameter holes that are tens-of-microns deep. This system can be easily integrated into commercial laser micromachining processes, and can be employed in laser drilling and three-dimensional active-feedback laser printing.
\end{abstract}

Keywords: laser machining; depth measurement; confocal structure

\section{Introduction}

Precision laser micromachining plays a key role in the biomedical, electronics, and material processing industries, providing high-precision control of drilling depth for blind holes and heterogeneous structures. Q-switched lasers, which are also known as giant pulse lasers, can deliver giant pulses for use in laser machining, with their ablation rate during drilling decreasing with hole depth [1]. The drilling rate is highly nonlinear with depth and machining time, due to a number of effects involving the laser drilling process, including laser defocusing, the production of laser-induced plasmas, and varying material specifications. Several inline and repeatable methods designed for real-time monitoring or in-situ measurements of the laser ablation depth have been reported but continue to have limitations. Currently, the standard industrial process is to adopt a trial-and-error approach to determine laser machining curves for each sample, which outline the drilling depth over time under specific laser conditions. However, during laser drilling, machining conditions may be affected by unpredicted circumstances, such as laser power decay, clearance of the focusing system, optical misalignments resulting from mechanical vibrations, and human action. High throughput and quality assurance are required during offline, high-samplingrate inspections, and are, therefore, costly. Indeed, many techniques for monitoring of laser ablation depth in real-time have been reported.

Optodynamical methods for monitoring laser micro-drilling have been proposed and developed by Yeack et al. [2]. One can verify that laser drilling occurs by simultaneously detecting the laser-induced ultrasonic waves in a workpiece and in its surrounding air caused by ablation [3]. Such methods can directly measure the hole depth using piezo electric pressure sensors [4] or a laser beam deflection probe (LBDP) [5] that measures the time-of-flight of the shock resulting from laser ablation. Use of a LBDP is implemented experimentally by means of a digital micrographic system that enables the acquisition of images of the plasma plume and the hole cross-section during a drilling sequence. Optodynamic techniques have been applied to determining ablation depth in situations where the time-of-flight of the acoustic shock wave originating from a pulse can be detected using piezoelectric or interferometric techniques as it exits the rear of a material. This 
approach is easy to set up and can be integrated with commercial laser machining systems. However, one challenge in using this method is that workpieces may be heterogeneously structured. A technique that uses open piezoelectric acoustic sensors placed above the workpiece has been developed, but the characteristics of the acoustic pulse are dependent on the material properties [5] making it unsuitable for industrial situations where differing or non-homogeneous materials are common. Another approach to the real-time monitoring of laser processing uses the current induced by an electric field applied to a laser-produced plasma [6]. The resulting design for a new electrode circuit has allowed for an increased probing distance between the electrodes and the processed sample surface, thereby avoiding the direct contact of the electrodes with the plasmas and reducing sputtering. Papazoglou and coworkers [7] demonstrated the depth-resolved analysis of multi-layer structures is carried out by employing laser-induced breakdown spectroscopy (LIBS) and white light interferometry, which enables accurate in-situ depth monitoring. On-line LIBS determination of magnesium coating thickness [8] was performed by Ruiz et al. Several optical distance-measurement techniques have been proposed and implemented, with nanometer precision and the multi-millimeter depth ranges required in laser micro-hole drilling. Direct distance measurement techniques utilizing an autofocusing probe using two cylindrical lenses for profile measurement [9] or pure interferometric techniques $[10,11]$ have been used to measure the distances required with great accuracy, and could be suitable for ablation depth measurement. Direct interferometric techniques have been used to measure micron and sub-micron distances in several applications [9-11], but such techniques rely on high-intensity, directly-reflected light to discern the interference fringes. This limitation has been overcome in optical coherent tomography [12], where a coherent confocal imaging system is used to determine distance [13]. However, this method lacks simplicity and can be difficult to integrate into an industrial setting. All optical techniques are of value in their particular applications, but are inadequately flexible when applied to detecting laser-generated micron-scale hole depths in difficult geometries.

In this work, we present an approach for the inline, precise monitoring of laser ablation depth as applied to blind-hole laser drilling for single and multilayer bulk heterogeneous structures, and for different materials, an alloy and a silicon wafer. The detection method was based on the reflection of the probe beams from the bottom surfaces of the drilled holes. Using theoretical modeling and practical use cases, this method can be implemented to control error to within $5 \%$ for $100-\mu \mathrm{m}$ hole depths.

\section{Materials and Methods}

The principle of confocal structure was described by Marvin Minsky in 1957 [14] and has been applied to many sophisticated laser-scanning systems designed for a range of biomedical applications. Major improvements to the instrumentation photon efficiency, coupled with the development of novel fluorescent reporters, have enabled the multidimensional imaging of living cells and tissues [15]. Confocal microscopy uses point illumination and a pinhole in an optically conjugate plane in front of the detector to filter out defocused image information. Only light emanating from the focal plane is detected, allowing image quality to be far superior to that of wide-field fluorescence images. More-over, the thickness of the focal plane is inversely proportional to the square of the numerical aperture of the objective lens used, and is dependent on the optical properties of the specimen and ambient index of refraction [12]. This model of confocal microscopy applies to the confocal structure of laser ablation depth detection. A confocal microscope only receives the signal reflected back from the focal plane of the objective in a specimen, a property of confocal systems that is implemented here. By scanning the pinhole, the position of maximum signal intensity can be found that corresponds to the depth of a hole's bottom surface being drilled during laser machining.

The method proposed here uses a probe laser beam to illuminate the workpiece with a confocal structure. The experimental scheme is shown in Figure 1. The confocal optical system was integrated with a commercial laser machining station. The machin- 
ing laser was a 1064-nm Q-switched $\mathrm{Nd}: \mathrm{YVO}_{4}$ laser. The probe laser was a continuous wave 50-mW 532-nm frequency-doubling $\mathrm{Nd}: \mathrm{YVO}_{4}$ laser. Its beam size was about $1 \mathrm{~mm}$ without focusing lens. The pulse repetition frequency was tunable from 1 to $120 \mathrm{kHz}$. A set of half-wave plates (HWP) and a polarizer (PL) were placed after the machining and probe lasers in order to allow for output power adjustment. A $45^{\circ}$ dichroic mirror (DM) was employed to combine the beams from the machining and probe lasers.

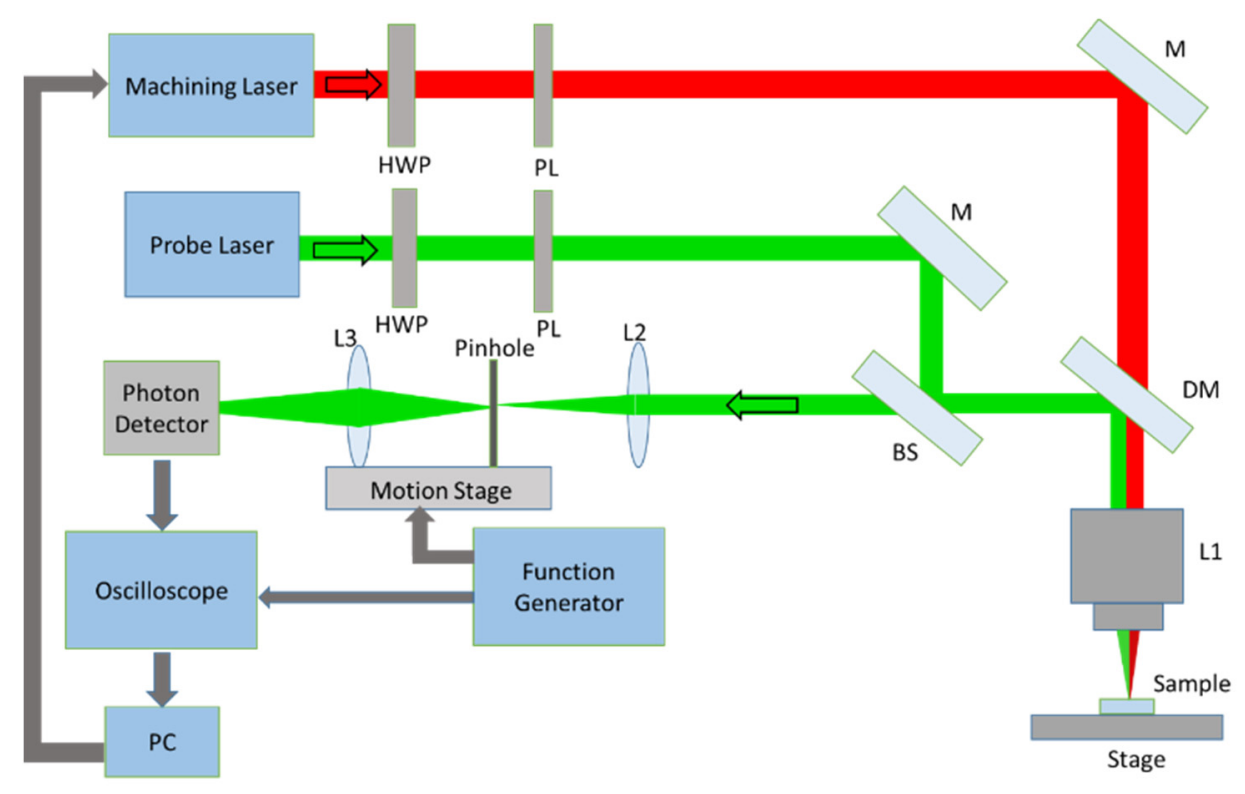

Figure 1. Schematic optical layout for real-time depth monitoring. HWP: Half-Wave Plate; PL: Polarizer; DM: Dichroic Mirror; BS: Beam Splitter; M: Mirror; and PC: Personal Computer.

The probe beam was reflected by a 50/50 beam splitter (BS) and a DM, focused on the workpiece by Lens 1 (L1), and then reflected back from the bottom surface of the ablated area of the workpiece where the beam was gathered by the same focusing lens. The quasi-collimated gathered light was then reflected by a DM, passed through a BS, and focused on a 10- $\mu \mathrm{m}$-diameter pinhole by another focusing lens (L2). The diffracted light after the pinhole was focused on a photo detector (PD) by a focusing lens (L3). The signals from the PD were sent to the oscilloscope connected to a computer. The pinhole and focusing lens (L3) were arranged on a linear motion stage (New Focus 9066, Newport, IR, USA) driven by an iPico module (New Focus 8763, Newport, IR, USA). The simultaneous search for the maximum intensity position on the PD while the line motional stage moved was driven by a function generator with triangle waveform. The laser drilling process on the samples typically induced the debris, nanosized dust, and even plasma. They may cause the fluctuation along the optical path and result in the measurement noises. So, we performed the measurements after the laser drilling processes. Each position scan of a linear motion stage costs $1.2 \mathrm{~s}$. Each measurement process was performed after the laser drilling process. And the 0.5 -s interval between them. The durations of data acquisitions and the depth calculations of the drilled hole was less than $0.1 \mathrm{~s}$. A complete drilling and measurement cost about $3 \mathrm{~s}$. Therefore, the operation time was still long for mass productions. Higher measurement accuracy for a pulse-to-pulse operation within $1 \mathrm{~s}$ was still studied.

Inserting a BS into the optical path of a machining laser can result in defocusing. This defocusing results in $z$-position shift of the focal point, and can be solved by finding a new $z$-axis distance. In short (ns) and ultrashort pulse (ps/fs) laser machining, pulse shape deformation may be induced by optical elements in the optical path [16]. Here, the BS thickness was $2 \mathrm{~mm}$ and the pulse duration of the machining laser was $10 \mathrm{~ns}$. After checking the pulse shape by a PD with a gigahertz response frequency, pulse shape deformation was monitored. 
To increase the position sensitivity, the focal lengths of Lenses 1 and 2 were chosen. We analyzed a theoretical model by numerically simulating two cases, and then compared the experimental data with the model.

\section{Theoretical Model Using Numerical Simulation}

A schematic of the test platform is shown in Figure 2a. The optical layout of Figure 1 was simplified as Figure 2a. The focal length of the machining laser focusing lens (L1) in the two cases was set to $50 \mathrm{~mm}$. For the confocal lens (L2), the focal length was set to $50 \mathrm{~mm}$ in Case 1, and $100 \mathrm{~mm}$ in Case 2. The distance (d) between lenses L1 and L2 was the sum of their focal lengths. The results of the simulation are presented in Figure $2 b$. In Case 1, the travel distance of the focusing lens shifted $1 \mathrm{~mm}$, and the intensity peak in the confocal setup shifted $1 \mathrm{~mm}$, yielding a lateral sensitivity ratio of 1:1 over $1 \mathrm{~mm}$ of travel. In Case 2, the travel distance of the focusing lens shifted $1 \mathrm{~mm}$, and the intensity peak in the confocal setup shifted $4 \mathrm{~mm}$, yielding a lateral sensitivity ratio of 1:4 over $1 \mathrm{~mm}$ of travel. Some degradation in the resolution of the system was observed due to mechanical vibration and imperfect optical alignment. The simulation works were performed with Matlab.

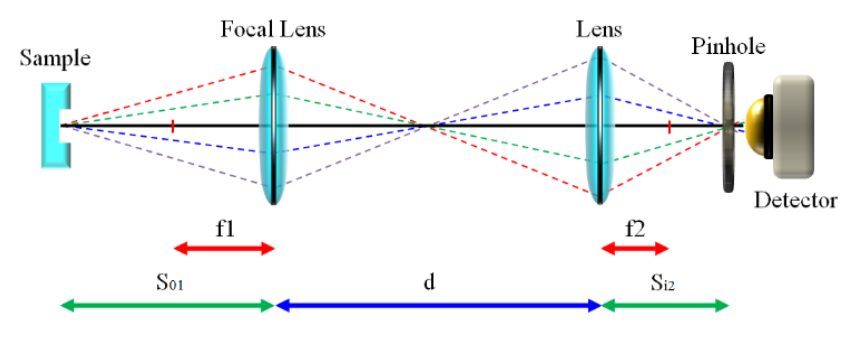

(a)

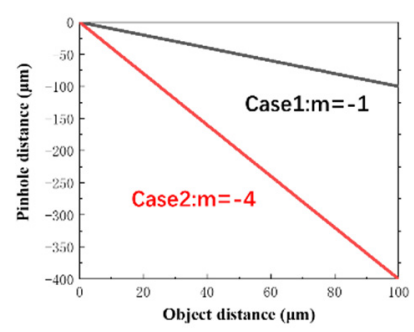

(b)

Figure 2. (a) Illustration of simplified confocal depth detection system; and (b) simulation results of the lateral sensitivity gain of 1:1 (blue line) and 1:4 (red line) over $100 \mu \mathrm{m}$ of travel, respectively. (Case 1: $\mathrm{f}_{1}=50 \mathrm{~mm}, \mathrm{f}_{2}=50 \mathrm{~mm}$; Case $2: \mathrm{f}_{1}=50 \mathrm{~mm}, \mathrm{f}_{2}=100 \mathrm{~mm}$ ).

\section{Results and Discussion}

\subsection{Confocal System Tested Using Polished Stainless Steel}

The optical system was configured to utilize a probe laser as previously described, and parameters were chosen as discussed in the numerical simulation section. A $10-\mu \mathrm{m}$ pinhole was utilized for the confocal element, and a confocal lens with a focal length of $100 \mathrm{~mm}$ and a suitable collecting lens were chosen to focus the light passing through the pinhole onto the PD.

A piece of polished stainless steel was first affixed to the laser workstation as a sample workpiece. Initially, the probe laser beam was aligned collinearly with the machining laser beam. The vertical stage was fine-tuned through a portion of its range to simulate the movement of the surface of the machined bottom of the drilled hole. The relation of the object distance and the pinhole distance using acquired data is plotted in Figure 3a. The sample was measured at different vertical positions in $20 \mu \mathrm{m}$ increments from 0 to $120 \mu \mathrm{m}$. The data obtained while scanning the object distance at varied vertical positions using Cases 1 and 2 are shown in Figure 3b,c, respectively. In Figure 3a, the root-mean-square (RMS) uncertainty of curve fitting for Case 1 was calculated as $0.046 \mu \mathrm{m}$. And, the RMS uncertainty of curve fitting for Case 2 was $0.025 \mu \mathrm{m}$. These experimental results confirm that, as indicated by the theoretical and numerical studies, the confocal mechanism for micron-precision hole depth detection effectively tracked the change in relative distance of the workpiece. In particular, as the workpiece moved away from the focus of the focusing lens, the signal intensity and signal-to-noise ratio decreased. 

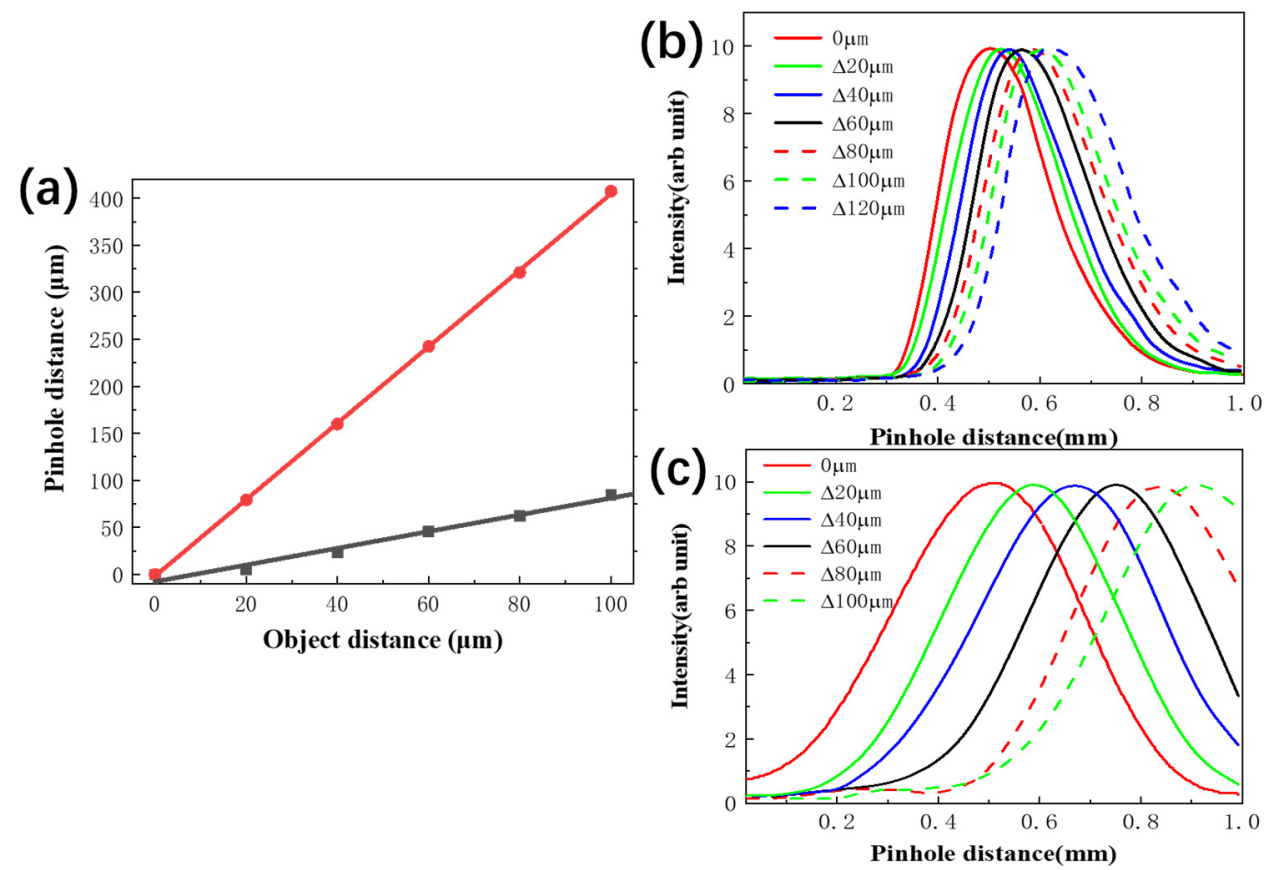

Figure 3. (a) Relation of the object distance and a pinhole distance, (b) data from Case 1, and (c) data from Case 2.

\subsection{The Designed Experiments for Laser Drilling in Aluminum Alloy}

A series of experiments was designed to showcase the capability of the system. After the alignment of the optics was checked, a small piece of aluminum (Al) alloy was cut and prepared, then affixed to the stage. Then, the Al alloy workpiece was drilled by $20-60$ pulses by focusing a 1064-nm pulsed laser with a 0.5-J pulse energy onto the workpiece. The pinhole was then scanned over its entire range of travel, and data were collected with the depthmonitoring system. After the sample was drilled and measured, a three-dimensional (3D) image map of the sample was obtained using a 3D laser microscope (Keyence VK-9700, Tokyo, Japan).

The surface of the $\mathrm{Al}$ alloy was drilled by 20 pulses with $0.5-\mathrm{J}$ pulse energies. Figure $4 \mathrm{a}$ shows a 3D image map from the laser microscope, with a cross-section included as Figure $4 \mathrm{~b}$. Figure $4 \mathrm{c}$, d show the scan data using Cases 1 and 2, respectively. The hole depth was measured to be $34.04 \mu \mathrm{m}$ using the 3D laser microscope. In Case 1, the hole depth was measured to be $35 \mu \mathrm{m}$ with the confocal optical design, with an error of $\sim 2.94 \%$. In Case 2, the position of the maximum intensity measured was $136 \mu \mathrm{m}$, so assuming the numerical simulation of the 4:1 ratio of transverse position, the hole was calculated to be $33.75 \mu \mathrm{m}$ deep with an error of $\sim 0.85 \%$. At different drilling depths, the number of machining laser pulses was increased to 40 and 60 pulses. These experimental data are shown in Figures 5 and 6, and summarized in Table 1. The experimental processes were performed more than three times, and the error was generated from the standard derivation of data sets. The more than $10 \%$ measurement variations occurred and when the hole depth was larger than $170 \mu \mathrm{m}$. 
(a)

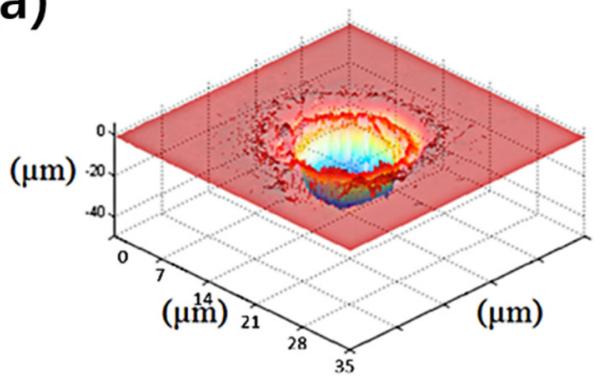

(c)

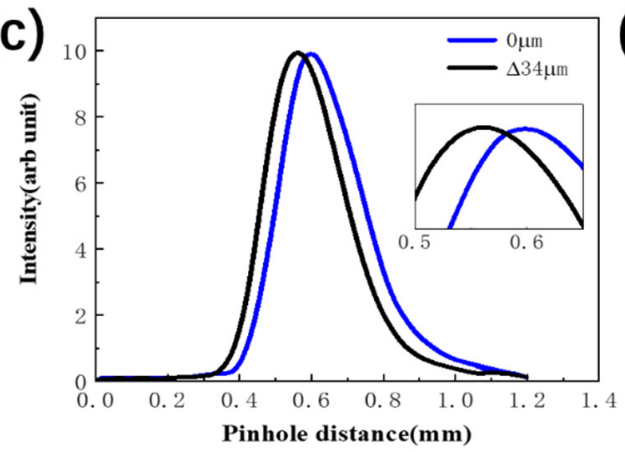

(b)
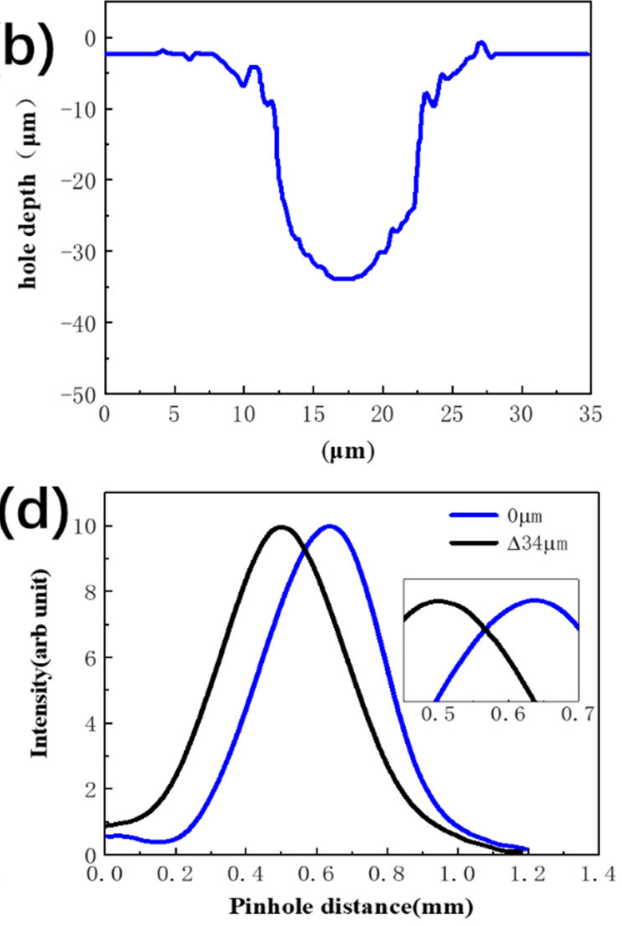

Figure 4. Image of the drilled hole after laser drilling with 20 pulses. (a) 3D confocal method; (b) cross-section; and (c,d) data from Cases 1 and 2, respectively.

(a)
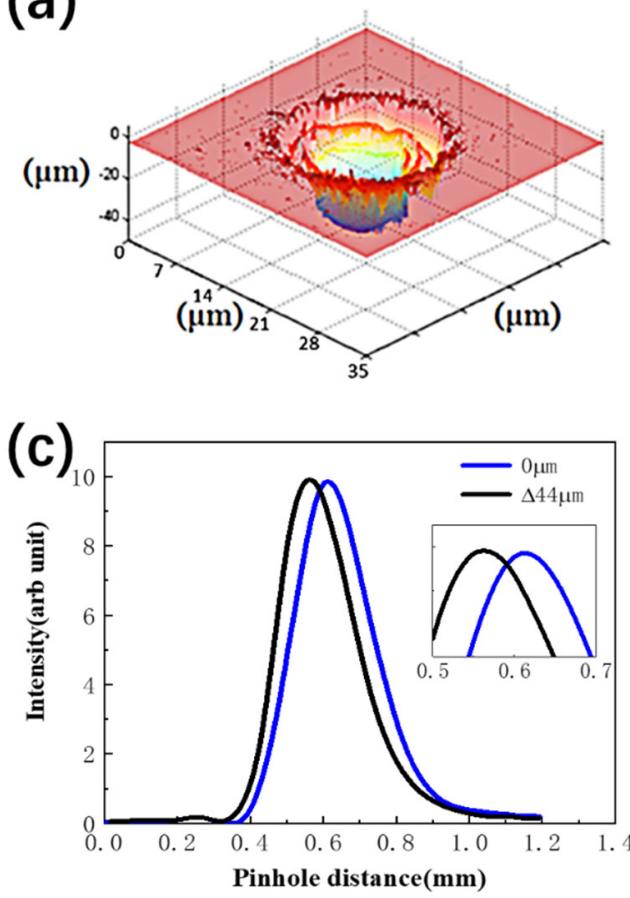

(b)

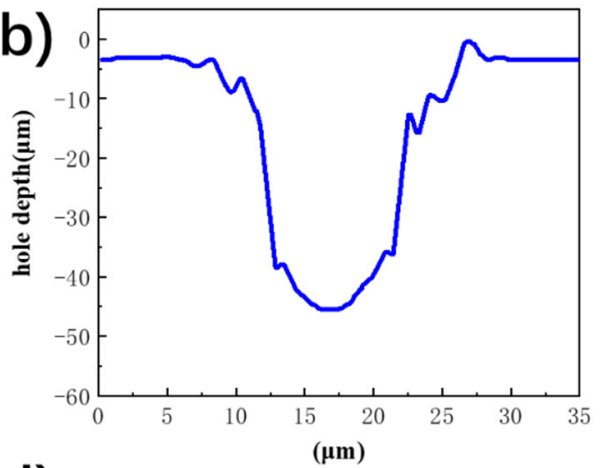

(d)

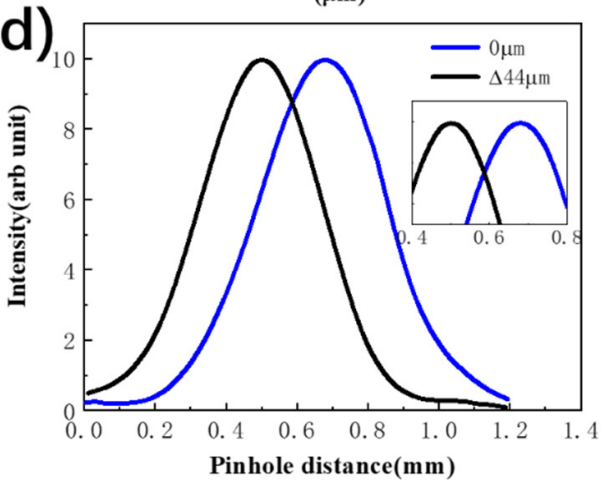

Figure 5. Image of the drilled hole after laser drilling with 40 pulses. (a) 3D confocal method; (b) cross-section; and (c,d) data from Cases 1 and 2, respectively. 
(a)
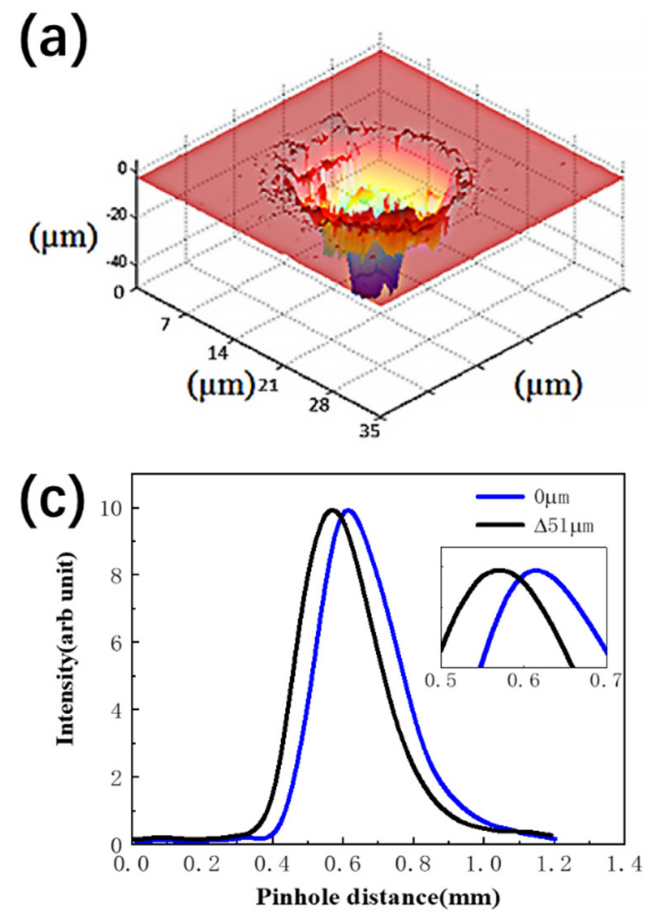

(b)
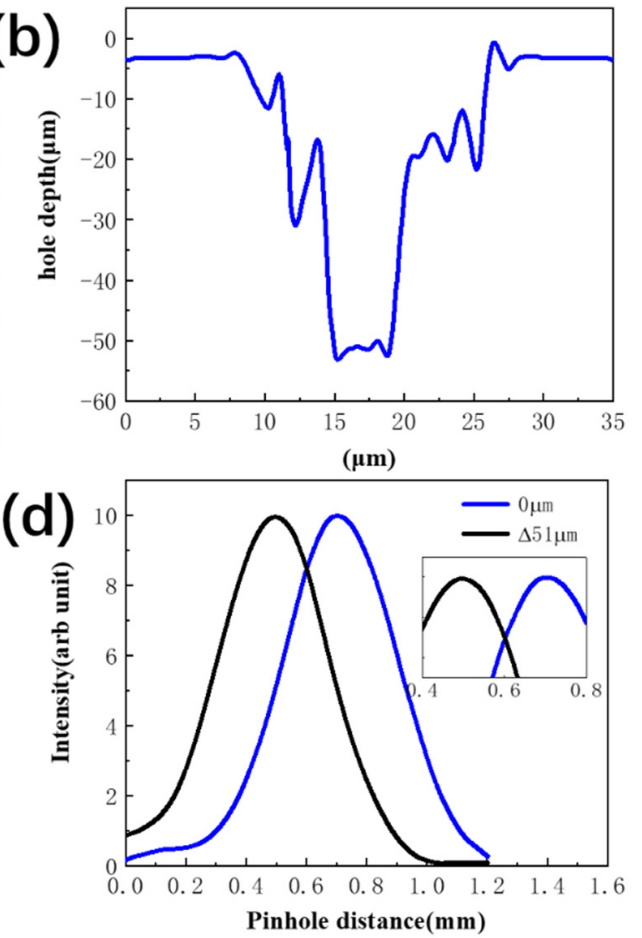

Figure 6. Image of the drilled hole after laser drilling for 60 pulses. (a) 3D confocal method; (b) crosssection; and (c,d) data from Cases 1 and 2, respectively.

Table 1. Summary of experimental data.

\begin{tabular}{cccc}
\hline \multirow{2}{*}{ Number of Laser Pulses } & \multicolumn{3}{c}{ Hole Depth and Error (Unit: $\boldsymbol{\mu m}(\%))$} \\
\cline { 2 - 4 } & 3D Laser Microscope & Case 1 & Case 2 \\
\hline 20 & 34.04 & $35(2.94 \%)$ & $33.75(0.85 \%)$ \\
40 & 44.81 & $45(0.44 \%)$ & $45.5(1.56 \%)$ \\
60 & 51.15 & $50.4(1.37 \%)$ & $53(3.71 \%)$ \\
\hline
\end{tabular}

\section{Conclusions}

This study performed theoretical simulations and experiments pertaining to the development of an all-optical real-time laser drilling depth detection method. The approach is based on a confocal optical structure, achieves a precision of $<1 \mu \mathrm{m}$ and an inaccuracy of $<5 \%$ for $\sim 100-\mu \mathrm{m}$-deep holes. This precision and accuracy are sufficient for real-time machining depth monitoring during laser micro-hole drilling, and the system is sufficiently robust for use in industrial and research applications. Furthermore, this method is independent of the wavelengths of the machining lasers, and can be integrated with commercial laser-machining systems in general. A reliable control process can be easily implemented for automatic control of machining depth in applications that are sensitive to the required depth of blind holes, eliminating the need for expensive back-wall protection, and highly-skilled and experienced operators. A $>100-\mu$ m-deep detections can be realized with a low-divergence-angle beam on the targeted sample surfaces. This study is still on-going.

Author Contributions: Conceptualization, X.C. and H.-C.C.; Data Measurement, Y.X. and S.S.; Data Analysis, Y.X. and N.-K.C.; Writing and Supervision, H.-C.C. All authors have read and agreed to the published version of the manuscript.

Funding: Ministry of Science and Technology, Taiwan (MOST 107-2622-E-006-014-CC3), and by the Fundamental Research Funds for the Central Universities, China (DUT18RC(3)047 and DUT20RC(5)028).

Institutional Review Board Statement: Not applicable. 
Informed Consent Statement: Not applicable.

Data Availability Statement: The data presented in this study are available in the article.

Conflicts of Interest: The authors declare no conflict of interest.

\section{References}

1. Petkovsek, R.; Panjan, I.; Babnik, A.; Mozina, J. Optodynamic study of multiple pulses micro drilling. Ultrasonics 2006, 44, E1191-E1194. [CrossRef]

2. Yeack, C.E.; Melcher, R.L.; Klauser, H.E. Transient photo-acoustic monitoring of pulsed laser drilling. Appl. Phys. Lett. 1982, 41, 1043-1044. [CrossRef]

3. Strgar, S.; Mozina, J. An optodynamic determination of the depth of laser-drilled holes by the simultaneous detection of ultrasonic waves in the air and in the workpiece. Ultrasonics 2002, 40, 791-795. [CrossRef]

4. Stafe, M.; Negutu, C.; Popescu, I.M. Real-time determination and controlof the laser-drilled holes depth. Shock Waves 2005, 14, 123-126. [CrossRef]

5. Petkovsek, R.; Babnik, A.; Diaci, J.; Mozina, J. Optodynamic monitoring of laser micro-drilling of glass by using a laser probe. Appl. Phys. A-Mater. Sci. Process. 2008, 93, 141-145. [CrossRef]

6. Idris, N.; Madjid, S.N.; Ramli, M.; Kurniawan, K.H.; Lee, Y.I.; Kagawa, K. Monitoring of laser processing using induced current under applied electric field on laser produced-plasma. J. Mater. Process. Technol. 2009, 209, 3009-3021. [CrossRef]

7. Papazoglou, D.G.; Papadakis, V.; Anglos, D. In situ interferometric depth and topography monitoring in LIBS elemental profiling of multi-layer structures. J. Anal. At. Spectrom. 2004, 19, 483-488. [CrossRef]

8. Ruiz, J.; González, A.; Cabalín, L.M.; Laserna, J.J. On-Line Laser-Induced Breakdown Spectroscopy Determination of Magnesium Coating Thickness on Electrolytically Galvanized Steel in Motion. Appl. Spectrosc. 2010, 64, 1342-1349. [CrossRef]

9. Rhee, H.G.; Kim, D.I.; Lee, Y.W. Realization and performance evaluation of high speed autofocusing for direct laser lithography. Rev. Sci. Instrum. 2009, 80, 073103. [CrossRef]

10. Lading, L.; DamHansen, C.; Rasmussen, E. Surface light scattering: Integrated technology and signal processing. Appl. Opt. 1997, 36, 7593-7600. [CrossRef]

11. Hofstetter, D.; Zappe, H.P.; Dandliker, R. Optical displacement measurement with GaAs/AlCaAs-based monolithically integrated Michelson interferometers. J. Lightwave Technol. 1997, 15, 663-670. [CrossRef]

12. Webb, R.H. Confocal optical microscopy. Rep. Prog. Phys. 1996, 59, 427-471. [CrossRef]

13. Arons, E.; Leith, E. Coherence confocal-imaging system for enhanced depth discrimination in transmitted light. Appl. Opt. 1996, 35, 2499-2506. [CrossRef] [PubMed]

14. Minsky, M. Memoir on inventing the confocal scanning microscope. Scanning 1988, 10, 128-138. [CrossRef]

15. Paddock, S. Over the rainbow: 25 years of confocal imaging. Biotechniques 2008, 44, 643-648. [CrossRef]

16. Tan, B. Deep micro hole drilling in a silicon substrate using multi-bursts of nanosecond UV laser pulses. J. Micromech. Microeng. 2006, 16, 109-112. [CrossRef] 